\title{
CALIDAD UNIVERSITARIA: EXPECTATIVAS DE LOS ESTUDIANTES RECIÉN INCORPORADOS
}

\author{
RUBÉN FERNÁNDEZ ORTIZ \\ Universidad de La Rioja, España \\ ruben.fernandez@dee.unirioja.es \\ LEONOR GONZÁLEZ MENORCA \\ Universidad de La Rioja, España \\ leonor.gonzalez@dee.unirioja.es \\ NICOLÁS FERNÁNDEZ LOSA \\ Universidad de La Rioja, España \\ nicolas.fernandez@dee.unirioja.es \\ MARÍA JOSÉ SEGURA ANDOLLO \\ Centro Educativo González Gallarza \\ Comunidad Autónoma de La Rioja, España \\ mjsabdollo@hotmail.com
}

\section{RESUMEN}

En la actualidad, la preocupación por la calidad en la educación universitaria es elevada. De hecho, todas las Universidades se centran en el énfasis en los estudiantes y en la satisfacción de sus necesidades, ya que hay factores del entorno que así lo exigen. Hay un estancamiento en la entrada de alumnos y una cierta competencia por captar nuevos estudiantes. En este trabajo se pretende analizar las expectativas de satisfacción de los estudiantes con sus titulaciones y conocer lo que esperan de la Universidad y, más concretamente, de la titulación en la que se han matriculado, siendo uno de los objetivos el conocer las competencias genéricas que demandan a la Universidad y las competencias más específicas por Facultades y Titulaciones.

PALABRAS CLAVES: ESPACIO EUROPEO DE EDUCACIÓN SUPERIOR, CALIDAD UNIVERSITARIA, SATISFACCIÓN DE LOS ESTUDIANTES, COMPETENCIAS.

\section{ABSTRACT}

Currently, there is a major concern about the quality of higher education. Most universities are impelled to organize themselves in relation to students' expectations and the satisfaction of their needs. Universities are fighting to counteract the decline of student populations and compete fiercely to attract new students. Students' expectations in relation to their programs are analyzed as well as their demands in terms of the general competences of the university, its schools and programs.

KEY WORDS: STUDENT SATISFACTION, EUROPEAN SPACE FOR HIGHER EDUCATION (ESHE), UNIVERSITY QUALITY, COMPETENCES

\section{INTRODUCCIÓN}

En el paradigma enseñanza-aprendizaje se está produciendo un cambio que subraya cada vez más la importancia de una educación centrada en el sujeto que aprende. Esto supone un desplazamiento de una educación centrada en la enseñanza hacia una educación centrada en el aprendizaje. El paradigma anterior implicaba un énfasis en la adquisición y transmisión del conocimiento. Los elementos para el cambio de dicho marco teórico actual incluyen: una educación más centrada en el estudiante, una transformación del papel del educador, una nueva definición de objetivos, un cambio en el enfoque de las actividades educativas, un desplazamiento de la importancia de los suministros de conocimientos a los resultados y un cambio drástico en la organización del apren- 
dizaje. Este punto de vista subraya que el estudiante, el que aprende, es el centro del proceso. Por consiguiente, afecta la manera de encauzar las actividades educativas y la organización del conocimiento, que pasan a ser regidos por las metas del estudiante (Proyecto Tuning, 2003).

¿Y cuáles son esas metas? ¿Cómo consideran nuestros estudiantes que debe ser su formación académica? ¿Qué valoran más, la cantidad y calidad de los conocimientos adquiridos, la adecuación de la formación adquirida en la Universidad para conseguir luego el puesto de trabajo deseado, el desarrollo de determinadas competencias mentales y emocionales, la posibilidad de que el título obtenido sea válido en otros países, que el profesorado de su Universidad le facilite todo lo anterior, que las infraestructuras y servicios de su Universidad se adecuen a sus necesidades?

En un reciente trabajo se desarrolló una escala de medida de la satisfacción de los estudiantes con el servicio universitario en el ámbito del Espacio Europeo de Educación Superior, y se identificaron los factores subyacentes que la conforman. Ahora se pretende analizar las expectativas de algunos de los principales usuarios directos del servicio, los estudiantes recién incorporados a la Universidad, determinando qué factores resultan más atractivos y pueden generar niveles más altos de satisfacción, estableciendo diferencias por Facultades y Titulaciones.

El interés de este trabajo radica en que resulta de imperiosa necesidad profundizar en el análisis de las expectativas y actitudes que tienen los estudiantes que acaban de incorporarse a la Universidad para que sirva de guía y contraste en las nuevas directrices y actuaciones que progresivamente se irán implantando en el inmediato futuro europeo universitario. En concreto, también servirá para establecer las competencias genéricas que demandan los estudiantes universitarios en general y las competencias específicas de cada una de las Facultades y Titulaciones.

\section{El alumno universitario: nuestro cliente}

No existe consenso sobre quiénes son o no clientes del sistema educativo universitario (Hill, 1995; Cuthbert, 1996; Made y Kuei, 1993; Owlia y Aspinwall, 1996) pero, tras la revisión bibliográfica, parece existir cierto consenso sobre que los estudiantes sí pueden ser considerados clientes. Cada vez hay mayor coincidencia en que la opinión de los estudiantes es fuente imprescindible para evaluar la enseñanza universitaria (Escudero, 2000). El alumno no se puede considerar como un mero comprador o receptor pasivo de un producto; es necesario considerar su participación en el proceso de enseñanza/aprendizaje (Hansen y Jackson, 1996). El énfasis en el alumno y en satisfacer sus necesidades puede parecer un planteamiento arriesgado si, como señalan algunos autores (Barret, 1996), el estudiante no sabe lo que quiere cuando entra en la Universidad, llegando incluso a considerar denigrante este objetivo. Hay Universidades que reconocen al estudiante como su principal cliente, como por ejemplo la Oregon State University que recoge, como uno de sus principios básicos, que "Ios alumnos son sus clientes más importantes". Todo ello, sin entrar en contradicciones con el hecho de que la Universidad tiene una misión primordial de servicio para con la sociedad y de asunción de responsabilidades científicas, culturales y sociales, que van más allá de la cobertura de las necesidades de los alumnos (García Garrido, 1997; Michavilla y Tabatoni, 1997).

En estos últimos años, son varios los trabajos que abogan por las ventajas de conocer lo que esperan los estudiantes cuando entran en la Universidad, su satisfacción y sus necesidades, con la finalidad de establecer indicadores no sólo objetivos sino también subjetivos y, de esta forma, poder tomar decisiones más eficientes. Este tipo de estudios puede ser debido a la influencia de los cambios en el entorno (distrito único, avance de las nuevas tecnologías, Espacio Europeo, 
entre otros) que obliga a las Universidades a ser más competitivas.

El estudiante a la hora de manifestar su grado de satisfacción con la Universidad va a tener en cuenta diversos factores (Rodríguez-Marín et al., 2002). Estos factores se han agrupado en cuatro bloques: aplicabilidad de los estudios, desarrollo de competencias, factores relacionados con la convergencia europea y organización de la Universidad.

Según el II Estudio sobre la Juventud Universitaria Española del BBVA, los estudiantes universitarios españoles se muestran satisfechos con la titulación que estudian y críticos con la inserción laboral que le ofrecen las instituciones académicas. La principal deficiencia que advierten los estudiantes es la baja formación práctica.

Se ha observado que en la actualidad el conocimiento que se valora en el ámbito laboral, se está desarrollando a un ritmo vertiginoso y este fenómeno hace que la formación que los estudiantes reciben en el aula quede obsoleta enseguida. Este hecho obliga a mantener relaciones constantes con el sector productivo. Pero, al mismo tiempo, es necesario un reajuste cualitativo y cuantitativo en el modelo de aprendizaje, cobrando valor aquello que contribuya al desarrollo intelectual de los alumnos y a incrementar la capacidad de adaptación a la diversidad y los cambios tecnológicos, económicos y culturales.

Por otra parte, hay que tener presente que existen diferencias en función de la titulación del estudiante. Los estudiantes de titulaciones que poseen mayor grado de experimentalidad otorgan más importancia relativa a las instalaciones y equipamientos, la cual se refleja en una expectativa más alta de utilización de los laboratorios y aulas informáticas, mientras que los estudiantes de Ciencias Empresariales valoran más el desarrollo de competencias mentales, probablemente por sus altas expectativas de inserción laboral en empresas de su entorno (Rodríguez-Marín et al., 2002; Capelleras, J. y Veciana, J. M., 2004).

Tras la fundamentación teórica anterior, en la que se manifiestan diferencias importantes entre las distintas carreras universitarias con respecto a la satisfacción del alumnado, se pretenden dos objetivos: 1) identificar el perfil de los alumnos de cada una de las Facultades y Titulaciones de la Universidad en función de la percepción y las expectativas que éstos tengan y 2) determinar la existencia o ausencia de relación entre la satisfacción del alumnado y las variables, para cada Facultad de forma individualizada, propuestas en el modelo general (Fernández et al., 2005) y las variables explicativas propuestas con base en la literatura.

En definitiva, se quiere estudiar, tomando aisladamente la muestra obtenida de cada Facultad, cuáles son los principales factores determinantes de la satisfacción de los alumnos. Con ello se busca especificar en mayor medida la valoración que, de los cuatro factores estudiados (aplicabilidad, competencias, organización y convergencia) (basado en el trabajo de Fernández et al. 2005) frente al concepto de satisfacción, hacen los alumnos de cada Facultad.

\section{DESCRIPCIÓN DEL ESTUDIO}

Esta sección se divide en dos apartados. En el primer apartado se presenta la metodología empleada en la investigación. El segundo apartado se centra en las variables del estudio.

\section{Metodología}

La calidad del servicio universitario ha sido medida a través de una escala de diferencial semántico. Se trata del instrumento más apropiado para valorar la percepción que tiene el cliente de los elementos fundamentales de la calidad del servicio (Trespalacios, Fernández y Díaz, 1999). La 
generación inicial de los ítems que finalmente compusieron el cuestionario se basó en la revisión de la literatura sobre calidad de servicio, calidad de la enseñanza y tendencias del Espacio Europeo de Educación Superior. Posteriormente, se entregó el cuestionario a varios colegas. Fruto de la consulta se decidió la eliminación de determinados ítems que resultaban ambiguos o no poseían suficiente información para su evaluación, así como la modificación de algunos de ellos.

La versión final del cuestionario quedó conformada por un total de 75 ítems estructurados en cuatro bloques ${ }^{1}$ con preguntas cerradas y codificados en una escala Likert de cuatro alternativas de respuesta:

1. búsqueda de una mayor empleabilidad y su conexión con la formación universitaria;

2. desarrollo de competencias genéricas y específicas;

3. reconocimiento académico y equivalencia;

4. nuevo rol docente y participación del estudiante en la estructura universitaria.

Este cuestionario se aplicó durante el mes de mayo del curso académico 2004-05, tras realizar un muestreo aleatorio estratificado, a una muestra de estudiantes de primer curso de todas las Facultades y Escuelas de la Universidad de La Rioja². Los investigadores se desplazaron a las distintas unidades estudiadas, donde se ofrecía a los estudiantes la oportunidad de contestar

1. Las dimensiones son: aplicabilidad, competencias, convergencia y organización interna.

2. El número de alumnos de nuevo ingreso por Facultades y Escuela son: Facultad de Ciencias Empresariales: 193; Facultad de Ciencias jurídicas y Sociales: 403; Facultad de Ciencias, Estudios Agroalimentarios e informática: 223; Facultad de Letras y de la Educación:744; Centros adscritos: 124 y Escuela Técnica Superior de Ingeniería Industrial:129. voluntariamente al cuestionario. Tras un proceso de validación, los cuestionarios finalmente utilizados fueron 429. Esta fase de obtención de los datos se realizó según se muestra en la ficha técnica de la tabla 1. Para que el estudiante pudiera emitir juicios relativos a los diversos aspectos que contenía el cuestionario, se escogió un momento avanzado del curso, de tal forma que hubiera tenido tiempo suficiente de formarse una opinión sólida en relación a todos estos.

Una vez codificada la información, se realizaron distintos tipos de análisis: 1) análisis de la validez y fiabilidad de la escala de medida; 2) medidas conjuntas de calidad del ajuste para el modelo de ecuaciones estructurales; 3) análisis factorial de componentes principales para identificar constructos teóricos que no pueden ser medidos directamente; 4) análisis factorial y análisis factorial confirmatorio de los distintos bloques del cuestionario; 5) análisis descriptivo de los factores explicativos de la satisfacción por Facultades y Titulaciones; 6) regresión lineal entre todos los factores y la satisfacción como variable dependiente (sólo por facultades); 7) regresión logística binomial; 8) análisis descriptivo de los doce factores identificados en el cuestionario con la variable dependiente binomial (satisfechos e insatisfechos).

TABLA 1

\section{FICHA TÉCNICA DE LA ENCUESTA}

\begin{tabular}{ll}
\hline Universo & $\begin{array}{l}\mathrm{N}=1816 \text { estudiantes de 1er. curso } \\
\text { de todas las Facultades y Escuelas } \\
\text { Universidad de La Rioja }\end{array}$ \\
\hline Ámbito & $\begin{array}{l}\mathrm{N}=429 \text { encuestas válidas. Índice } \\
\text { de respuesta }=23,6 \%\end{array}$ \\
\hline Nivel de confianza & $95 \% ; p=q=0,5$ \\
\hline Diseño muestral & Muestreo aleatorio estratificado \\
\hline Cuestionario & $\begin{array}{l}\text { Cuestionario estructurado en } \\
\text { Cuatro bloques con preguntas cerradas }\end{array}$ \\
\hline Fecha trabajo de campo & Mayo 2007 \\
\hline Tratamiento de los datos & SPSS 15.0, AM0S 8.0 \\
\hline
\end{tabular}




\section{Variables objeto del estudio}

El estudio de las dimensiones que configuran la satisfacción de los alumnos en el ámbito de la enseñanza universitaria se llevó a cabo mediante la aplicación de un análisis factorial y posteriormente, de una confirmación del mismo mediante ecuaciones estructurales. Luego, se llevó a cabo la interpretación de las dimensiones obtenidas, que quedan agrupadas de la siguiente forma:

\section{Bloque 1.-Aplicabilidad}

- Factor 1: realización de prácticas internas: en qué medida el número y calidad de los ejercicios prácticos, casos, entre otros., es el adecuado y sirve para clarificar la teoría.

- Factor 2: incorporación al mercado laboral: en qué medida los conocimientos adquiridos y destrezas desarrolladas durante su estancia en la Universidad le van a permitir encontrar un trabajo ajustado a su titulación y en un corto espacio de tiempo.

- Factor 3: realización de prácticas externas: en qué medida la realización de prácticas en empresas es importante para adquirir las capacidades y destrezas de su titulación.

- Factor 4: cercanía entre mercado laboral y Universidad: en qué medida existe una escasa distancia entre la formación que adquirirá en la Universidad y la requerida en el mercado laboral.

\section{Bloque 2.-Competencias}

- Factor 1: competencias mentales de autogestión: en qué medida la formación recibida en la Universidad le ayudará a mejorar su habilidad para planificar, programar trabajos, organizar tiempos, resolver problemas, aprender autónomamente, entre otros.
- Factor 2: competencias mentales de relación: en qué medida, la formación recibida en la Universidad le ayudará a mejorar su capacidad de hablar en público, su capacidad de toma de decisiones, su capacidad de relación con el entorno o su adaptabilidad.

- Factor 3: competencias emocionales de autogestión: en qué medida la formación recibida en la Universidad le ayudará a mejorar su capacidad de autocrítica, compromiso ético, capacidad de trabajo en situaciones de presión, entre otros.

- Factor 4: competencias emocionales de relación: en qué medida la formación recibida en la Universidad le ayudará a mejorar su capacidad de trabajo en equipo, sus relaciones interpersonales, su capacidad de transmitir conocimientos o su sensibilización social.

\section{Bloque 3.-Convergencia}

- Factor 1: movilidad: en qué medida valora positivamente la posibilidad de programas de movilidad interuniversitaria, realización de estancias en el extranjero o que el título obtenido sea reconocido a nivel europeo.

- Factor 2: nuevos métodos de aprendizaje: en qué medida valora positivamente el uso de métodos de enseñanza que requieran una responsabilidad del estudiante más elevada en su propio aprendizaje.

\section{Bloque 4.-Organización interna}

- Factor 1: profesorado: en qué medida el personal docente hace que el contenido de la asignatura sea estimulante, prepara bien las clases, motiva, utiliza métodos de enseñanza que requieren del estudiante pensar por sí mismo, entre otros. 
- Factor 2: infraestructuras: en qué medida los servicios internos de la Universidad son adecuados con respecto a gestiones administrativas, resolución de problemas, horarios; así como, el conocimiento por parte del alumno de todos los servicios e infraestructuras universitarias.

\section{Grado de satisfacción del estudiante descripción del estudio: valoración y expectativas}

Es básico para las Universidades definir su calidad a través de la identificación de lo que perciben sus estudiantes y de las expectativas que tienen. Puede que la Universidad alcance un alto nivel en algunas características y un bajo nivel en otras e incluso puede que una mejora en una de las dimensiones implique una reducción en otras.

Los resultados obtenidos en las Facultades y Escuela Politécnica son presentados en los anexos 1 y 2, los cuales son analizados a continuación.

\section{Bloque 1.-Aplicabilidad}

Factor 1: realización de prácticas internas (Apli1): El rango de la valoración media por parte del alumnado va de 2,74 hasta 3,25. Los estudiantes que en mayor medida consideran que debería incrementarse el número de realización de casos prácticos, problemas, entre otros., son aquellos matriculados en la Facultad de Ciencias, Estudios Agroalimentarios e Informática. Por el contrario, los alumnos que consideran que el componente práctico de sus asignaturas es adecuado son los pertenecientes a los Centros adscritos. Existen diferencias significativas entre la valoración media del alumnado, percibiéndose una mayor necesidad de realización de prácticas en las Facultades de Ciencias
Empresariales, en la de Letras y de la Educación, junto con la ya referida de Ciencias y Estudios Agroalimentarios e Informática. Sin embargo, la Escuela Técnica Superior de Ingeniería Industrial muestra una valoración superior a la media. Merece la pena mencionar que las titulaciones de Ingeniería Técnica en Hortofruticultura, Magisterio Infantil e Ingeniería Técnica Agraria son percibidas por el alumno como excesivamente teóricas. Por el contrario, las que tienen una mayor carga de prácticas en el primer año son Matemáticas, Turismo y Enfermería.

Factor 2: incorporación al mercado laboral (Apli2):

El rango de la valoración media por parte del alumnado va de 2,0781 hasta 2,9714. Los estudiantes que, en mayor medida, consideran que encontrarán un trabajo ajustado a las competencias y conocimientos adquiridos son los matriculados en la Escuela Técnica Superior de Ingeniería Industrial, en concreto en las titulaciones de Electrónica. Aquellos estudiantes más pesimistas son los pertenecientes a la Facultad de Ciencias, Estudios Agroalimentarios e Informática, además de las titulaciones de Químicas e Ingeniería Técnica Agrícola, especialidad Hortofruticultura.

Factor 3: realización de prácticas externas (Apli3): El rango va de 3,30 hasta 3,89, superando todas las Facultades, excepto la Facultad de Ciencias Jurídicas Humanas y Sociales la media de la valoración de la escala. Con respecto a las titulaciones, los alumnos de Filología Inglesa son aquellos que consideran en mayor medida la no necesidad de incrementar las prácticas externas; en el otro extremo se encontraría la titulación de Filología Hispánica donde sus alumnos mayoritariamente desean un mayor número de prácticas y valoración académica de las mismas. 
Factor 4: cercanía entre mercado laboral y Universidad (Apli4): Es el factor de aplicabilidad que mayor desviación típica ofrece, de 2,41 a 2,96. Se observa que, de una manera muy rotunda, son los alumnos de Filología Inglesa, ITI Electrónica y Turismo, quienes perciben una elevada cercanía de sus estudios al mercado laboral. Se encuentra que son las titulaciones de Enología, Matemáticas y Empresariales las que muestran una mayor distancia entre los contenidos ofrecidos y lo que piensa el alumno que está demandando el mercado laboral.

\section{Bloque 2.-Competencias}

Factor 1: competencias mentales de autogestión (H1): El rango de la valoración media por parte del alumnado ha ido de 2,90 hasta 3,22 . Los estudiantes que, en mayor medida, consideran que la formación recibida en la Universidad les capacita para planificar y programar trabajos, resolver problemas, organizar tiempos $y$, en definitiva, aprender autónomamente, son los de la Escuela Técnica Superior de Ingeniería Industrial. Por el contrario, los alumnos matriculados en la Facultad de Ciencias Empresariales consideran que la formación que reciben no contribuye a desarrollar suficientemente sus competencias mentales de autogestión. Existen diferencias significativas entre la valoración media del alumnado, percibiéndose una mayor necesidad de fomentar las competencias mentales de autogestión en la Facultad de Ciencias y Estudios Agroalimentarios e Informática, Facultad de Letras y Educación así como la mencionada de Empresariales.

Merece la pena mencionar que las titulaciones de IngenieríaTécnica en Hortofruticultura, Diplomatura en Empresariales y Licenciatura en Enología son percibidas por el alumnado como titulaciones que no contribuyen a la formación de competencias de autogestión. Por el contrario, los que perciben que la formación recibida en la Universidad les ayudará a planificar y programar trabajos, tiempos, entre otros. son Matemáticas, Filología Hispánica y Relaciones Laborales.

Factor 2: competencias mentales de relación (H2): El rango de la valoración media por parte del alumnado va de 2,7597 hasta 3,4105. Los estudiantes que en mayor medida consideran que la formación universitaria les ayudará a mejorar su capacidad de hablar en público, a tomar decisiones, entre otros, es decir, sus competencias mentales de relación, son los matriculados en los Centros Adscritos, en concreto, las titulaciones de Turismo y Relaciones Laborales. Aquellos estudiantes más pesimistas en este sentido, son los pertenecientes a la Facultad de Ciencias, Estudios Agroalimentarios e Informática y, en concreto, las titulaciones de Enología e ITA Hortofruticultura.

Factor 3: competencias emocionales de autogestión (H3): El rango va de 2,99 a 3,42, superando todas las Facultades, excepto la Facultad de Ciencias Empresariales, la media de la valoración de la escala. En concreto por titulaciones, los alumnos de la Diplomatura de Empresariales son aquellos que tienen menos confianza en que la formación recibida en la Universidad les vaya a ayudar a desarrollar su capacidad de autocrítica y su capacidad de trabajo ante situaciones de presión. En el otro extremo se encontraría la Licenciatura de Matemáticas, en la que sus alumnos mayoritariamente creen que la Universidad les va a formar en competencias emocionales de autogestión. 
Factor 4: competencias emocionales de relación (H4): Es el factor competencial que mayor desviación típica ofrece, de 2,85 a 3,55. Se observa que, de una manera muy rotunda, son los alumnos de Turismo, Enfermería y Magisterio Educación Física quienes mayor valoración hacen de la formación recibida en relación con su capacidad de trabajo en equipo, mejora de la transmisión de conocimientos, mejora de las relaciones interpersonales, entre otros., afectando así a los Centros Adscritos y a la Facultad de Letras y de la Educación. Se encuentra que son las titulaciones de Empresariales, Enología y Filología Inglesa las que muestran un mayor pesimismo sobre la previsible formación en competencias emocionales de relación.

\section{Bloque 3.-Convergencia}

Factor 1: movilidad (Converg. 1): El rango de la valoración media va de 3,49 a 3,89. Los estudiantes que, en mayor medida, valoran de manera positiva los programas de movilidad interuniversitaria o realización de estancias en el extranjero o en otras universidades españolas son los correspondientes a los Centros Adscritos. Por el contrario, los alumnos que menos interés muestran por estas actividades universitarias son los pertenecientes a la Facultad de Ciencias, Estudios Agroalimentarios e Informática, siendo la titulación de ITA Hortofruticultura la que presenta una menor valoración en este Centro.
Factor 2: nuevos métodos de aprendizaje (Converg.2): El rango va de 2,93 a 3,45. Los alumnos que, en mayor grado, desean adquirir más responsabilidad sobre su propio aprendizaje son los correspondientes a la Facultad de Letras y de la Educación, mientras que los que menos desean ser los protagonistas de su propio aprendizaje son los alumnos de la Facultad de Ciencias Empresariales y, especialmente, los alumnos de la Diplomatura de Empresariales.

\section{Bloque 4.-Organización interna}

Factor1: Profesorado (Oprof): Los alumnos de la Facultad de Ciencias Jurídicas y Sociales son los que mejor valoración expresan sobre su personal docente, siendo los alumnos de Ciencias Empresariales los que peor valoran esta dimensión.

Factor2: Infraestructuras (Oinfra): En este factor el rango va de 2,48 a 2,83, no existiendo demasiada desviación. La Facultad que mejor valora este aspecto es la de Ciencias Sociales y la peor la de Ciencias, Estudios Agroalimentarios e Informática, siendo la titulación de ITA Hortofruticultura la que da una puntuación menor.

En cuanto a las diferencias en las dimensiones que discriminan entre satisfacción y no satisfacción por Facultades, los resultados muestran lo siguiente:

\section{Variables en la ecuación: Escuela Técnica Superior de Ingeniería Industrial}

\begin{tabular}{|c|c|c|c|c|c|c|c|}
\hline Variable & B & S.E. & Wald & $d f$ & Sig & $\mathrm{R}$ & $\operatorname{Exp}(B)$ \\
\hline $\mathrm{H} 2$ & 2,8232 & ,6140 & 21,1435 & 1 &, 0000 & ,3401 & 16,8313 \\
\hline OPROF & 1,3027 & 6475 & 4,0479 & 1 & ,0442 & ,1112 & 3,6792 \\
\hline APLI2 & 1,1350 &, 5072 & 5,0076 & 1 &, 0252 & 1348 & 3,1113 \\
\hline
\end{tabular}


Variables en la ecuación: Facultad de Ciencias Empresariales

\begin{tabular}{|c|c|c|c|c|c|c|c|}
\hline Variable & B & S.E. & Wald & $d f$ & Sig & $\mathrm{R}$ & $\operatorname{Exp}(B)$ \\
\hline $\mathrm{H} 2$ & 2,8232 & ,6140 & 21,1435 & 1 & ,0000 & ,3401 & 16,8313 \\
\hline OPROF & 1,3027 & ,6475 & 4,0479 & 1 & ,0442 & ,1112 & 3,6792 \\
\hline APLI2 & 1,1350 &, 5072 & 5,0076 & 1 & , 0252 & ,1348 & 3,1113 \\
\hline
\end{tabular}

Variables en la ecuación: Facultad de Ciencias Jurídicas y Sociales

\begin{tabular}{cccccccc}
\hline Variable & B & S.E. & Wald & df & Sig & R & Exp(B) \\
\hline H2 & 3,1820 & 1,3809 & 5,3100 & 1 &, 0212 &, 3091 & 24,0953 \\
\hline
\end{tabular}

$\begin{array}{llllll}\text { Constant } & -9,8867 & 4,4843 & 4,8608 & 1 & , 0275\end{array}$

\begin{tabular}{cccccccc}
\multicolumn{6}{c}{ Variables en la ecuación: Facultad de Ciencias, Estudios Agroalimentarios e Informática } \\
Variable & B & S.E. & Wald & df & Sig & R & Exp(B) \\
\hline H2 & 4,4384 & 1,2558 & 12,4910 & 1 &, 0004 &, 3382 & 84,6436 \\
H4 & 1,5112 &, 8135 & 3,4511 & 1 &, 0632 &, 1258 & 4,5324 \\
CONVERG2 & $-1,3753$ &, 6153 & 4,9961 & 1 &, 0254 &,- 1807 &, 2528 \\
OPROF & 2,2684 &, 8452 & 7,2029 & 1 &, 0073 &, 2382 & 9,6637 \\
\hline
\end{tabular}

Constant $\quad-21,8098 \quad 5,2587 \quad 17,2010 \quad 1 \quad 0000$

Variables en la ecuación: Facultad de Letras y de la Educación

\begin{tabular}{cccccccc}
\hline Variable & B & S.E. & Wald & df & Sig & R & $\operatorname{Exp(B)}$ \\
\hline H1 & 2,6077 & 1,2864 & 4,1094 & 1 &, 0426 &, 1206 & 13,5680 \\
H2 & 3,4999 & 1,2951 & 7,3026 & 1 &, 0069 &, 1913 & 33,1115 \\
H3 & 1,8299 &, 7661 & 5,7052 & 1 &, 0169 &, 1599 & 6,2333 \\
ALPI1 & 3,3429 &, 9515 & 12,3444 & 1 &, 0004 &, 2672 & 28,3012 \\
\hline
\end{tabular}

$\begin{array}{llllll}\text { Constant } & -32,4920 & 7,5493 & 18,5244 & 1 & , 0000\end{array}$ 
Variables en la ecuación: Centros Adscritos

\begin{tabular}{cccccccc}
\hline Variable & B & S.E. & Wald & df & Sig & R & Exp(B) \\
\hline H2 & 3,3589 & 1,0105 & 11,0491 & 1 &, 0009 &, 2965 & 28,7568 \\
OPROF & 2,3151 &, 8505 & 7,4092 & 1 &, 0065 &, 2292 & 10,1256 \\
\hline
\end{tabular}

$\begin{array}{lllllll}\text { Constant } & -16,9008 & 4,1514 & 16,5741 & 1 & 0000\end{array}$

Como se observa, es el factor competencias mentales de relación la variable que discrimina entre satisfacción y no satisfacción en la Facultad de Ciencias Jurídicas y Sociales.

En el caso de la Facultad de Ciencias Empresariales son los factores competencias mentales de relación, profesorado y mercado laboral las variables discriminatorias. Esto quiere decir que, desde la Facultad, hay que hacer un esfuerzo por preparar a los estudiantes en aquellas competencias que les ayuden a mejorar su capacidad de relacionarse y adaptarse al entorno, en adaptar los contenidos y destrezas a la demanda laboral y que para ello, los profesores impartan sus enseñanzas a través de métodos docentes innovadores que ayuden a los estudiantes a pensar por sí mismos.

En el caso de la Escuela Técnica Superior de Ingeniería Industrial las variables discriminatorias son las mismas que las obtenidas en los alumnos de la Facultad de Ciencias Empresariales: competencias mentales de relación, profesorado y mercado laboral.

Al analizar los datos obtenidos en la Facultad de Ciencias, Estudios Agroalimentarios e Informática, se comprueba que aparece una dimensión nueva y son los novedosos métodos de aprendizaje, que incluyen tareas y actividades que vayan encaminadas a que sea el alumno quien se responsabilice de su aprendizaje.

Llama la atención en la Facultad de Letras y de la Educación, que no aparezca el factor profeso- rado siendo los factores que hacen referencia a las competencias los que sirven para discriminar. Lo que buscan los estudiantes de esta Facultad es que la formación que se les imparte les ayude a mejorar habilidades de planificación y programación, a interrelacionarse con el entorno y a autogestionar sus emociones. Asimismo, que se realicen suficientes casos y problemas como para clarificar la teoría.

En los Centros Adscritos, se comprueba cómo las dimensiones que se refieren a las competencias mentales de relación y la de profesorado son las que más se repiten y en las que, por lo tanto, la Universidad debería hacer un mayor esfuerzo en valorarlas y adoptar medidas que contribuyan a aumentar la satisfacción de los estudiantes.

\section{CONCLUSIONES}

Las principales conclusiones del presente trabajo, siempre sobre la base de un marco teórico y empírico, se pueden centrar en las siguientes:

1. Se ha desarrollado, evaluado y aplicado una escala para medir la valoración que los alumnos de nuevo ingreso en la Universidad $^{3}$ otorgan a los factores de aplicabilidad de los conocimientos adquiridos al mercado de trabajo, competencias por desarrollar, convergencia con Europa y organización interna de la propia universidad.

3. Al finalizar su primer curso académico en la Universidad. 
2. Se han analizado los resultados por Facultades y se han encontrado aquellas variables que sirven para discriminar entre satisfacción y no satisfacción de los estudiantes en los diferentes Centros, poniéndose de manifiesto la especificidad del ámbito de los estudios en cuanto a los resultados obtenidos.

Hay dos factores recurrentes en casi todas las Facultades: profesorado y competencias mentales de relación. Se da mucha importancia al hecho de que el profesorado haga que el contenido de la asignatura sea estimulante, prepare bien las clases, motive, utilice métodos de enseñanza que exigen al estudiante pensar por sí mismo, entre otros. Al mismo tiempo, se considera imprescindible que la formación recibida en la Universidad ayude al estudiante a mejorar su ca- pacidad de hablar en público, su capacidad de toma de decisiones, su capacidad de relación con el entorno o su adaptabilidad al mercado laboral.

Se considera que los resultados obtenidos en este tipo estudio pueden servir a los gestores de la Universidad para tomar decisiones, ya que estos muestran dónde radican las principales expectativas de los estudiantes y hasta qué punto se están cumpliendo en las diferentes Facultades. De esta forma, será también más fácil elaborar la estrategia a seguir para mejorar la calidad. Hay que tener en cuenta que actualmente las Universidades se están viendo afectadas por un entorno altamente competitivo y es necesario que funcionen con criterios de eficacia y de satisfacción hacia el estudiante y hacia la sociedad.

\section{ANEXO 1}

\section{RESULTADOS POR TITULACIONES ${ }^{4}$}

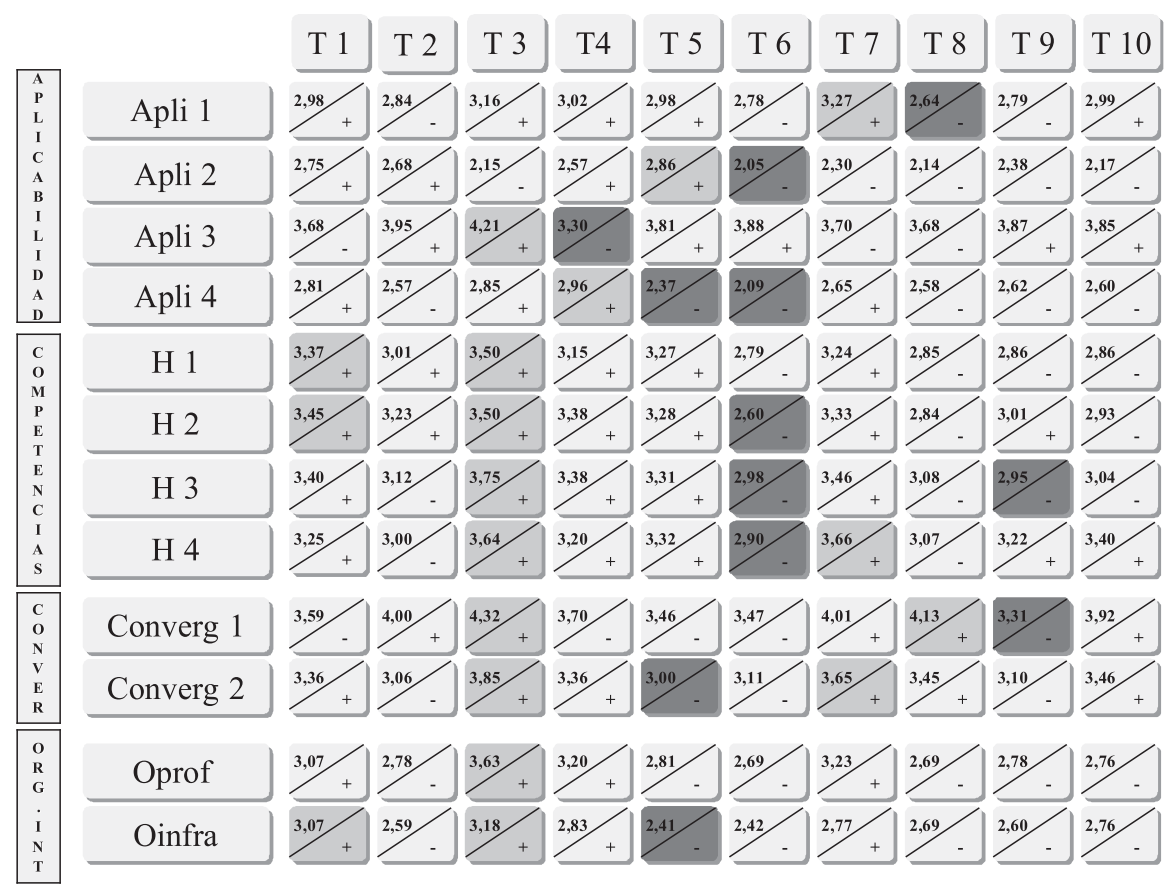

4. T1:Relaciones Laborales; T2: LADE;T3: Filología Hispánica;T4: Derecho; T5: ITI Eléctricos; T6:Enología;T7:Magisterio Educación Física; T8: Magisterio Educación Infantil;T9: Magisterio Educación Musical;T10: Magisterio Educación Lengua Extranjera; T11: Filología Inglesa; T12:Químicas; T13: Diplomatura en Ciencias Empresariales; T14:ITI Electrónica; T15: Matemáticas; T16: ITA Hortofrutícola; T17: ITA Industrias Agrarias; T18: Enfermería;T19:Turismo 


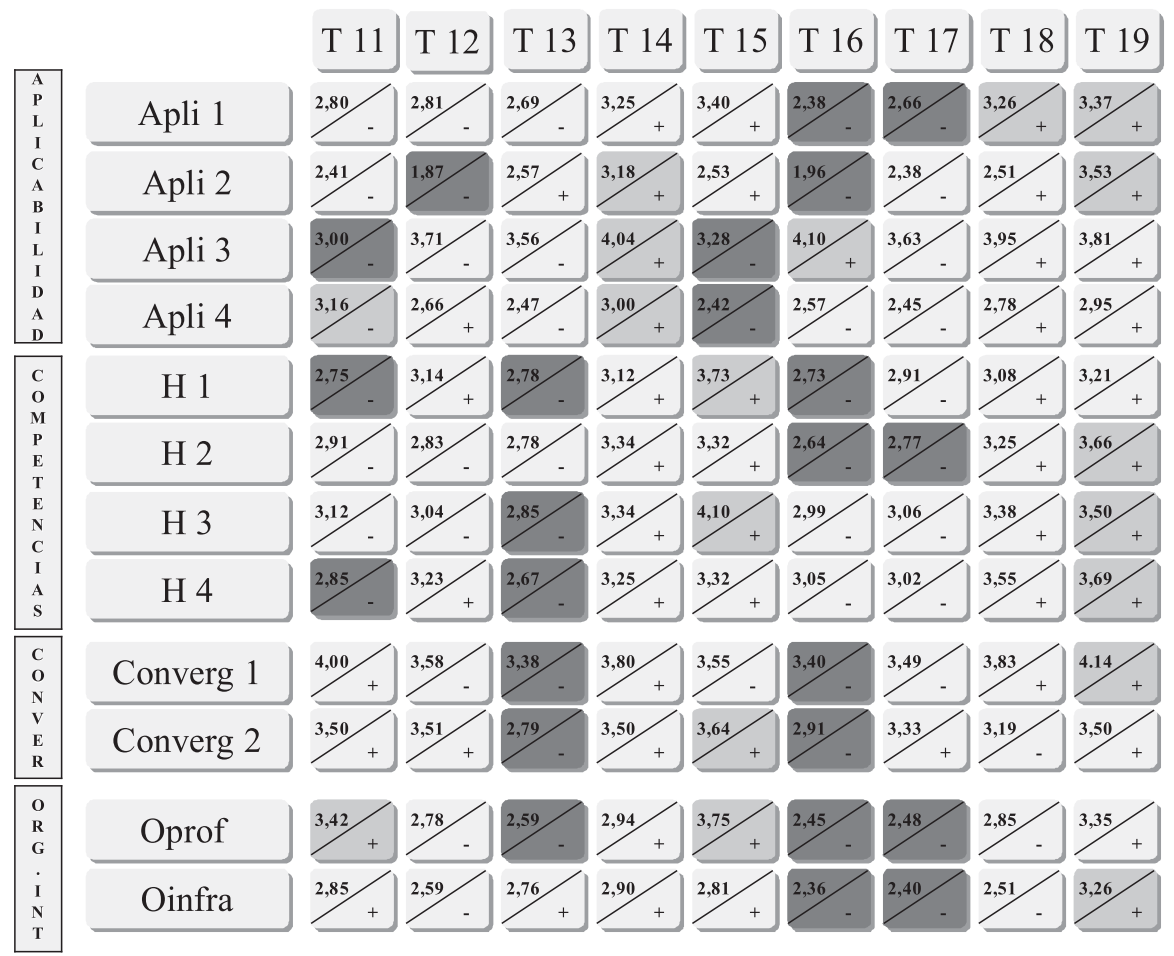

TABLA 3

\section{RESULTADOS POR FACULTADES 5}

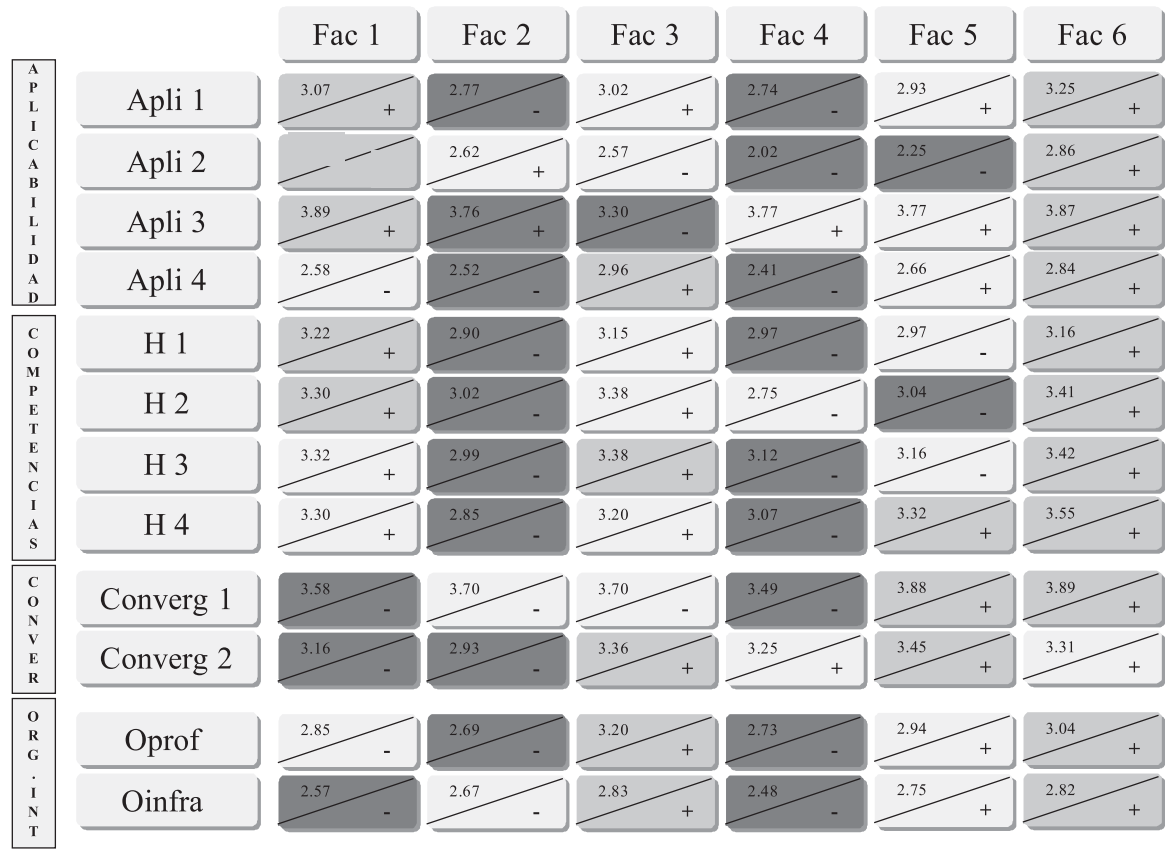

5. Fac.1: ETS de Ingeniería Industrial; Fac.2: Fac.Ciencias Empresariales; Fac.3: Fac. de Ciencias Jurídicas y Sociales; Fac.4: Fac. de Ciencias, Estudios Agroalimentarios e Informática; Fac.5: Facultad de Letras y de la Educación; Fac.6: Centros Adscritos. 


\section{REFERENCIAS}

Álvarez, V. \& Lázaro, A. (coord.) (2002). Calidad de las universidades y orientación universitaria. Archidona (Malaga). España: Aljibe.

Argudín, Y. (2000). La Educación Superior para el siglo XXI. Didac, 36, 16-25

Barnett, R. (1997). Towards a higher education for a new century. Institute of Education, University of London.

Barnett, R. (2001). Los límites de la competencia. El conocimiento, la educación superior y la sociedad. España: Gedisa.

Barret, R. (1996). Quality and the abolition of standards: arguments against some American prescriptions for the improvement of higher education. Quality in Higher Education, 2(3).

Batllori, R. \& Falgás, M. (2005). Expectativas de los estudiantes de universidad. VIII Symposium Internacional sobre las prácticas en empresas en la formación universitaria.

Bautista Vallejo, J. M. (2004). La Universidad y su espacio europeo en la encrucijada de la calidad.

Bricall, J. M. (2000). Informe Universidad 2000. CRUE

Camisón, C.; Gil, M. T. \& Roca, V. (1999). Hacia modelos de calidad de servicio orientados al cliente en las universidades públicas: el caso de la Universidad Jaume I. Investigaciones Europeas 5 (2).

Cuthbert, P. (1996). Managing service quality in HE: is servqual the answer? Part1. Management Service Quality, $6(2)$.

Dalgeggio, P. (2001). Profil de l'étudiant universitaire. Service d'Aide a l'Enseignement: Université de Montreal.

De Ketele, J. M. (2005). Las prácticas profesionales en la formación universitaria. VIII Symposium Internacional sobre las prácticas en empresas en la formación universitaria.

Dearing, R. (1997). Higher Education in the Learning Society: Report of the National Committe of Inquiry into Higher Education. España: HMSO and NCIHE Pub.

DECLARACIÓN DE BOLONIA (1999). El Espacio Europeo de la Educación Superior.

DECLARACIÓN DE LA SORBONA (1998). Declaración conjunta para la armonización del diseño del Sistema de Educación Superior Europeo.
Fernández, N., Fernández-Ortiz, R. \& González, L. (2005). The European space for higher education, students and university quality. XIV Congreso Internacional de la Academia Europea de Dirección y Economía de la Empresa: Simultaneidad Decisional y Multifocalidad Empresarial, 607-620.

Forner, A. (1996). Los futuros maestros. Cuadernos de Pedagogía, 247.

Gadea, A. (1999). Gestión de la calidad en los servicios públicos. La perspectiva de los ciudadanos, clientes y usuarios. España: Cuadernos IRC.

García Garrido, J. L. (1997). Tendencias de los sistemas educativos de cara al siglo XXI. Ciclo de Educación: una incógnita dentro del mundo actual, Universidad de Deusto.

Gil, I. (1995). La conceptualización y evaluación de la calidad de servicio al cliente percibida en el punto de venta. Club Gestión de Calidad.

González Sanmamed, M. (1995). Formación Docente: perspectivas desde el desarrollo del conocimiento y la socialización profesional. PPU.

González, J. \& Wagenaar, R. (2003). Tuning Educational Structures in Europe. Informe Final, Fase 1: Universidad de Deusto.

Heywood, L. (1993). Guide to development of competencebased standards for professions. Australian Government Publishing Service.

Joseph, M. \& Joseph, B. (1997). Service Quality in Education: A student perspective. Quality Assurance in Education, $5(1)$.

Latiesa, M. (1992). La deserción universitaria. Centro de Investigaciones Sociológicas.

Le Boterf, G., Barzuchetti, S. \& Vicent, F. (1993). Cómo gestionar la calidad de la formación. Gestión 2000.

Lemus Segura, L. (1996). Evaluación de la calidad de la Facultad de Educación de la Universidad Complutense de Madrid. UCM.

MECD (2003). La Integración del Sistema Universitario Español en el Espacio Europeo de Enseñanza Superior. Documento-Marco.

Micavilla, F. \& Tabotini, P. (1997). Alternativas de mejroa y evaluación institucional. Jornada Internacional Universidad y Calidad: Valores, Estategias y Gestión de la calidad en la Universidad. Universidad Ramón Llull. 
Molina, S. (2001). La universidad democráticamente masificada. Mira Editores.

Oldfield, B. M. \& Baron, S. (2000). Student perceptions of service quality in a U.K.. University Business and Management Faculty. Quality Assurance in Education, 8(2).

Oliver, R. (1980). A cognitive model of the antecedents and consequences of satisfaction decisions. Journal of Marketing Research, 17.

Parasuraman, A., Zeithaml, V. \& Berry, L. (1994). Reassessment of expectations as a comparison standard in measuring service quality: implications for further research. Journal of Marketing, 58.

Rodríguez-Marín, J., Mira, J. J., Pérez, V., García, A., Blaya, I. \& Martínez, B. (2002). Medición de las expectativas de los estudiantes universitarios: una herramienta para la planificación de la calidad. Psicología Social Aplicada, 13(3).

Tomás, M., Armengol, C. \& Feixas, M. (1999). Estudio de los ámbitos del cambio de cultura en la docencia universitaria. III Congreso de Innovación Educativa, Innovación en la Universidad. Universidad Santiago de Compostela.

Trespalacios, J. A., Fernández, M. \&Díaz, A. M. (1999). Calidad de la formación dirigida al comercio minorista: desarrollo y validación de una escala de medida. Cuadernos de Economía y Dirección de la Empresa, 4, 301-324.

Tunnermann, C. (1996). A new vision of higher education. Higher Education Policy, 9 (1), 11-27.

Watts, A. G. \& Esbroeck, V. (2005). New Skills for New Futures. Higher Education Guidance and Counselling Services in the European Union.

Recibido: $14-06-2010$ Aceptado: 29-09-2010 